\title{
EXCITATION OF ELECTRON WAVE PACKETS IN LOW-DIMENSIONAL SEMICONDUCTOR STRUCTURES BY INHOMOGENEOUS LIGHT PULSES
}

\author{
P. KRÁL AND J. MAŠEK
}

Institute of Physics, Na Slovance 2, 18040 Praha, Czecho-Slovakia

Generation of electron wave packets by space inhomogeneous light pulses is studied in low-dimensional semiconductor systems. Examination of their free propagation for ultrashort times as well as in the stationary excitation regime is performed. Non-classical effects related to the inhomogeneity of the source are predicted.

PACS numbers: $72.90 .+y, 78.90 .+t$

\section{Introduction}

Recent theoretical and experimental works in the mesoscopic physics [1] have tested the use of the elementary quantum mechanics in solids. It is now possible to study the collisionless (ballistic) electron transport in low-dimensional semiconductor systems [2]. The time-of-flight experiments have been broadly employed [3], but several problems of a practical origin should be treated more carefully. An example is the generation of electrons in these structures, performed recently by tunneling and optical injections.

We suggest a new generation mechanism by laser light pulses focused on micron spots. The excitation process is investigated in a time domain for short as well as long times of excitation. The free evolution of the wave packets in $1 \mathrm{D}$ and $2 \mathrm{D}$ electron gas (ballistic regime) is also examined.

\section{Model description}

We consider that the exciting laser beam, focused on microns spots, has a moderate strength $E=10^{6}-10^{8} \mathrm{~V} / \mathrm{m}$. Then the system is probably not far from equillibrium. The pulse is described in the plane of the sample by the effective vector potential $\boldsymbol{A}(\boldsymbol{x}, t)$ with the Gaussian profile

$$
A(x, t)=\left[A(t) \sin \left(\omega_{l} t\right) \exp \left(-x^{2} / 2 \sigma_{x}^{2}-y^{2} / 2 \sigma_{y}^{2}\right), 0,0\right] \text {. }
$$


The vector potential $\boldsymbol{A}(\boldsymbol{x}, t)$ of frequency $\omega_{l}$ is modulated by a rectangular time envelope $A(t)$ of the length $t_{p}$, ranging in a subpicosecond region. The space widths of the beam $\sigma_{x, y}$ are of the order of one micron.

In $1 \mathrm{D}$ and $2 \mathrm{D}$ the semiconductor system is modeled by a two parabolic band model with the effective masses $m_{\mathrm{e}}^{*}$ and $m_{\mathrm{v}}^{*}$ for the bulk GaAs. The electrons interacting with the light are described in the Coulomb gauge and the Bloch wave basis by the Hamiltonian

$$
\begin{aligned}
H= & \sum_{i=\mathrm{c}, \mathrm{v}} E_{k_{i}} a_{k_{i}}^{+} a_{k_{i}}+\sum_{k_{\mathrm{c}}, k_{\mathrm{v}}}\left[k_{\mathrm{v} x} \mathcal{A}^{*}\left(k_{\mathrm{v}}-k_{\mathrm{c}}, t\right) a_{k_{\mathrm{c}}}^{+} a_{k_{\mathrm{v}}}\right. \\
& \left.+k_{\mathrm{c} x} \mathcal{A}\left(k_{\mathrm{v}}-k_{\mathrm{c}}, t\right) a_{k_{\mathrm{c}}}^{+} a_{k_{\mathrm{v}}}\right]
\end{aligned}
$$

where the most important driving terms have been included. Here $\mathcal{A}(k, t)$ is the Fourier transform of $A(x, t)$.

We use the first order of the time perturbation theory to explore the perturbed wave functions for band electrons. From them the one-electron density matrix projected on the conduction band is constructed

$$
\begin{aligned}
& \left.\rho\left(k_{\mathrm{c}}, k_{\mathrm{c}}{ }^{\prime}, t\right)=\left(\frac{A}{2 m_{\mathrm{e}}^{*}}\right)^{2} \exp \left[-\mathrm{i} t\left(E_{k_{\mathrm{c}}}-E_{k_{\mathrm{c}^{\prime}}}\right) / 2 \hbar\right] k_{\mathrm{c} x} k_{c_{x}}{ }^{\prime} \sum_{k_{\mathrm{v}}} \mathcal{A}^{*}\left(k_{\mathrm{v}}-k_{\mathrm{c}}\right)\right] \\
& \times \mathcal{A}\left(k_{\mathrm{v}}-k_{\mathrm{c}}{ }^{\prime}\right) \frac{\sin \left[t\left(E_{k_{\mathrm{c}}}-E_{k_{\mathrm{v}}}-\hbar \omega_{l}\right) / 2 \hbar\right]}{\left(E_{k_{\mathrm{c}}}-E_{k_{\mathrm{v}}}-\hbar \omega_{l}\right) / 2 \hbar} \frac{\sin \left[t\left(E_{k_{\mathrm{c}}}-E_{k_{\mathrm{v}}}-\hbar \omega_{l}\right) / 2 \hbar\right]}{\left(E_{k_{\mathrm{c}}}-E_{k_{\mathrm{v}}}-\hbar \omega_{l}\right) / 2 \hbar} .
\end{aligned}
$$

The expression (3) is the golden rule in the case of non-homogeneous excitation ( $k$-momentum is not conserved). It can be easily transformed into the mixed coordinates one-electron Wigner distributions $\rho_{\mathrm{Wig}}(k, x, t)$.

\section{Examples}

The photoexcited electron distribution, Eq. (3), was examined in various physical situations. In Fig. 1 there is the 1D case for several times of excitation $t_{p}=0.2 \mathrm{ps}(\mathrm{a})$ and $t_{p}=35 \mathrm{ps}(\mathrm{b})$. The electrons with $k$-vectors around $k_{R}=$ $\pm k_{\text {res }}$ are excited. Two important effects are related with the local nature of the excitation:

1) For short excitation times $t_{p}$ the width $\Delta k$ of the electron $k$-spectrum and the energy width $\Delta E$, related to it through the dispersion relations, are given by the Heisenberg uncertainty relations

$$
\Delta k=\frac{4 \pi m_{\mathrm{e}}^{*} m_{\mathrm{v}}^{*}}{\hbar t_{p} k_{\mathrm{res}}\left(m_{\mathrm{e}}^{*}+m_{\mathrm{v}}^{*}\right)}, \quad \Delta E=\frac{\hbar}{m_{\mathrm{e}}^{*} k_{\mathrm{res}}^{2} \Delta k} .
$$

For longer $t_{p}$ the spectral width $\Delta k$ saturates to finite values (see Fig. 1b).

$$
\Delta k_{\lim }=\frac{\pi m_{\mathrm{e}}^{*}}{\sigma\left(m_{\mathrm{e}}^{*}+m_{\mathrm{v}}^{*}\right)}, \quad \Delta E_{l i m}=\frac{\hbar}{m_{\mathrm{e}}^{*} k_{\mathrm{res}}^{2} \Delta k_{\mathrm{lim}}} .
$$

Notice that $\Delta k_{\lim }$ is less than the geometrical spectral width $\Delta k_{\mathrm{g}}=\pi / 2 \sigma$ for $m_{\mathrm{e}}^{*}<m_{\mathrm{v}}^{*}$.

2) For short times of excitation $t_{p}$ the Wigner distribution $\rho_{\mathrm{Wig}}$ is positively definite, while for longer $t_{p}$ it has non-classical overshoots to negative values. For very long $t_{p}$ (see Fig. 1b) these overshoots appear only at the margins of $\rho_{\mathrm{Wig}}$. 

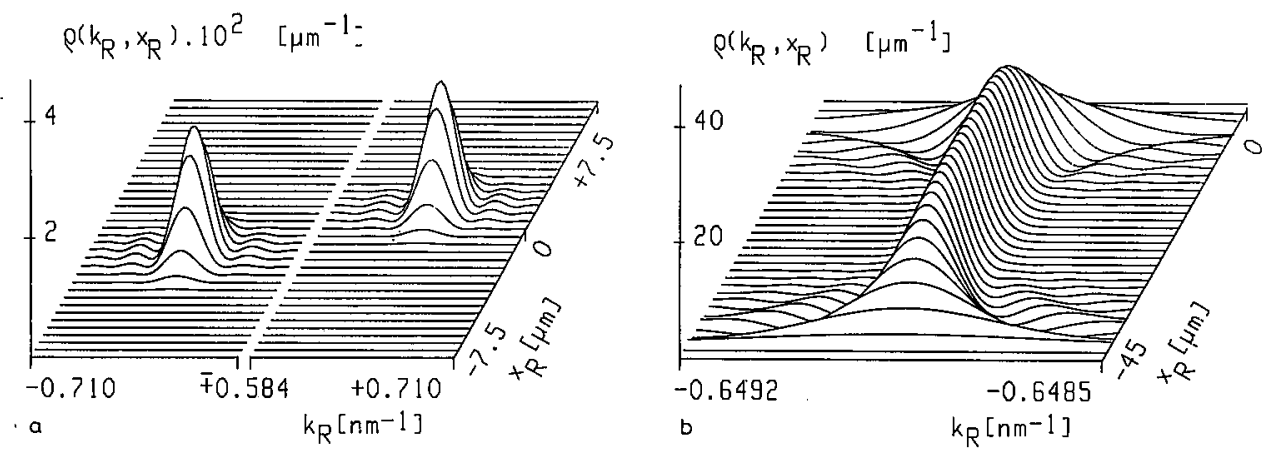

Fig. 1. The Wigner distribution in $1 \mathrm{D}$ is presented for several times of excitation. It is formed by two wave packets moving in opposite directions and located at two $k_{R}$. vectors: $k_{R}= \pm k_{\text {res }}$. For short excitation times $t_{p}=200$ fs in Fig. 1a characteristic oscillations from golden rule appear in $\rho$ Wig. But for longer excitation times non-classical negative overshoots appear at the space ends of $\rho_{\text {Wig }}$. These overshoots survive in the asymptotical excitation process, seen in Fig. $1 \mathrm{~b}$, where $t_{p}=35$ ps. The distribution has some limit width $\Delta k_{\text {lim }}$.
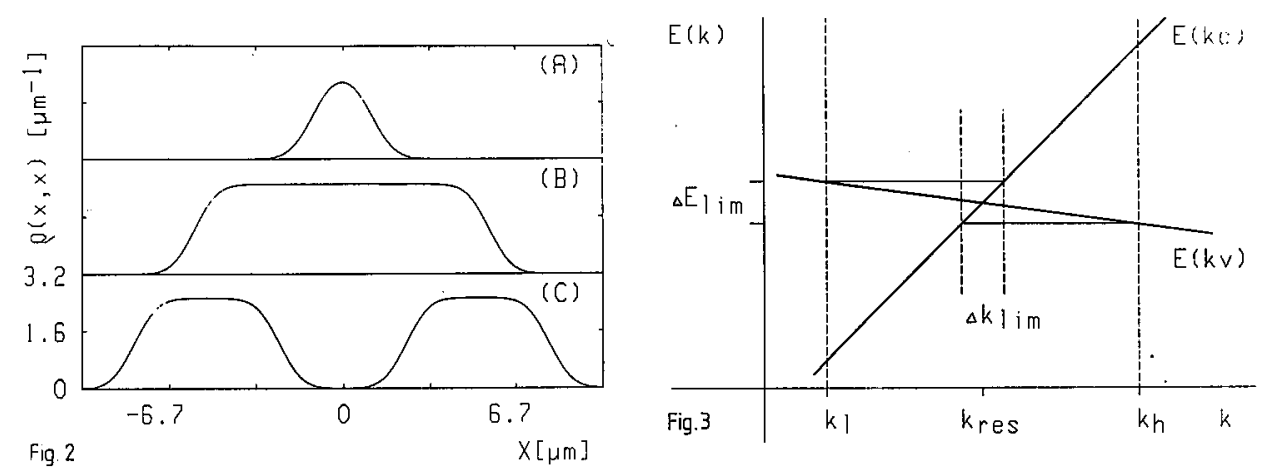

Fig. 2. The charge distributions for $\rho_{\mathrm{Wig}}$ in $1 \mathrm{D}$ are presented. In cases (A) $t_{p}=1$ ps and (B) $t_{p}=5 \mathrm{ps}$. For longer times (B) of excitation the distribution $\rho(x, x)$ saturates to finite value, corresponding to the given magnitude of the light field. In case (C) the evolution of the distribution from (B) for $t_{f}=3 \mathrm{ps}$ is shown.

Fig. 3. Band scheme for the excitation: the valence band shifted for $\hbar \omega_{l}$ crosses the conduction band. The light excites the electrons from the interval $\left\langle k_{l}, k_{h}\right\rangle$ in the valence band to a narrower interval in the conduction band. This leads to the non-classical negative oscillations in $\rho_{\text {Wig }}$.

In Fig. 2 there are the charge distributions for $\rho_{\text {Wig }}$ from Fig. 1. In (A,B) there is $t_{p}=1,5 \mathrm{ps}$ and in the case (C) the evolution of the distribution from (B) for $t_{f}=3 \mathrm{ps}$ is shown. The excited conduction electrons move ballistically from the excitation spot with the group velocity $v_{\mathrm{g}}=\hbar k_{\mathrm{res}} / m_{\mathrm{e}}^{*} \sim 1[\mathrm{~nm} / \mathrm{fs}]$. The resulting current densities look in the same way, but they are odd functions of $x$. 
Figure 3 shows the band scheme relevant for the excitation process. The valence band is shifted by $\hbar \omega$ and hybridized with the conduction band locally in the iluminated region. In the limit of long excitations, $t_{p} \rightarrow \infty$, the energy is conserved but momentum is not. The transitions are "horizontal". The dashed lines define the intervals in the Brillouin zone in which the excitation from valence to conduction band proceeds. The intervals have different widths because of different slopes of the dispersion relations for $\mathrm{v}$ - and c-band. The wider interval ( $\mathrm{v}$-band in case $m_{\mathrm{e}}^{*}<m_{\mathrm{v}}^{*}$ ) equals to the geometrical spectral width $\Delta k_{\mathrm{g}}=k_{h}-k_{l}=\pi / 2 \sigma$. The narrower interval (c-band in present case) equals to $\Delta k_{\text {lim }}$ from Eq. (5). This compression below the geometrical spectral width leads to the non-classical oscillations of the Wigner distribution.

In the stationary regime the electrons can be approximately described by the mixed Gaussian distribution

$$
\left.\rho_{\text {non equ. }} \sim \sum_{k_{\mathrm{c}}} \mid k_{\mathrm{c}}\right) \exp \left[-8\left|k_{\mathrm{c}}-k_{\text {res }}\right|^{2} / \Delta k_{\mathrm{lim}}^{2}\right]\left\langle k_{\mathrm{c}}\right| .
$$

For this distribution we can use in a close analogy with optical beams [4] the concept of a coherence length

$$
L_{\text {coh }}=2 \pi / \Delta k_{\text {lim }} \text {. }
$$

Electrons in the distribution (beam) are correlated up to the distance $L_{\mathrm{coh}}$.

\section{Conclusion}

We have described the formation and propagation of the electron wave packets in the early stage of the excitation as well as in the stationary excitation regime. The width of the energy spectrum of the electron wave packets have been investigated in details.

For longer pulses non-classical oscillations in the Wigner distributions $\rho_{\text {Wig }}$ are predicted if $m_{\mathrm{e}}^{*}<m_{\mathrm{v}}^{*}$. This is a general feature independent of the sample dimension, exact in the weak-field limit.

The spectral width $\Delta k$ saturates for longer excitation times to its asymptotical value $\Delta k_{\text {lim }}$. We find a simple law, which relates $\Delta k_{\text {lim }}$ to the diameter of the laser spot and the effective masses of the sample. In this case the concept of the coherence length of the electron beam can be introduced having a similar meaning as in the optical beam theory.

Two spot excitations in 2D were also studied. In the direction parralel to the two spots the two electron beams appear uncorrelated. This is because the correlation length for excited valence electrons is $\sigma$, so that valence electrons in different spots are not correlated. The same must be true for the conduction electrons. In the orthogonal direction the two spots behave as two Young slits.

\section{Acknowledgment}

One of the authors (P.K.) would like to thank Dr. B. Velický for introduction to the problem and Dr. P. Lipavský for fruitful discusions. 


\section{References}

[1] see reviews in: IBM J. Res. Dev. 32 (1988).

[2] B.J. van Wees, H. van Houten, C.W.J. Beenakker, J.G. Williamson, L.P. Kouwenhoven, D. van der Marel, C.T. Foxon, Phys. Rev. Lett. 60, 848 (1988).

[3] L. Reggiani, in: Physics of Non-Linear Transport in Semiconductors, Ed. P. Beaumont, Plenum Press, New York 1980, p. 243.

[4] J. Peřina, Coherence of Light, Van Nostrand, London 1972. 